\title{
A novel protein-repellent dental composite containing 2-methacryloyloxyethyl phosphorylcholine
}

\author{
Ning Zhang ${ }^{1,2}$, Chen Chen ${ }^{1,3}$, Mary AS Melo ${ }^{1}$, Yu-Xing Bai ${ }^{2}$, Lei Cheng ${ }^{3}$ and Hockin HK Xu ${ }^{1,4,5,6}$
}

Secondary caries due to biofilm acids is a primary cause of dental composite restoration failure. To date, there have been no reports of dental composites that can repel protein adsorption and inhibit bacteria attachment. The objectives of this study were to develop a protein-repellent dental composite by incorporating 2-methacryloyloxyethyl phosphorylcholine (MPC) and to investigate for the first time the effects of MPC mass fraction on protein adsorption, bacteria attachment, biofilm growth, and mechanical properties. Composites were synthesized with 0 (control), $0.75 \%, 1.5 \%, 2.25 \%, 3 \%, 4.5 \%$ and $6 \%$ of MPC by mass. A commercial composite was also tested as a control. Mechanical properties were measured in three-point flexure. Protein adsorption onto the composite was determined by the microbicinchoninic acid method. A human saliva microcosm biofilm model was used. Early attachment at $4 \mathrm{~h}$, biofilm at 2 days, live/dead staining and colony-forming units (CFUs) of biofilms grown on the composites were investigated. Composites with MPC of up to $3 \%$ had mechanical properties similar to those without MPC and those of the commercial control, whereas $4.5 \%$ and $6 \%$ MPC decreased the mechanical properties $(P<0.05)$. Increasing MPC from 0 to $3 \%$ reduced the protein adsorption on composites $(P<0.05)$. The composite with $3 \%$ MPC had protein adsorption that was $1 / 12$ that of the control $(P<0.05)$. Oral bacteria early attachment and biofilm growth were also greatly reduced on the composite with $3 \%$ MPC, compared to the control $(P<0.05)$. In conclusion, incorporation of MPC into composites at $3 \%$ greatly reduced protein adsorption, bacteria attachment and biofilm CFUs, without compromising mechanical properties. Protein-repellent composites could help to repel bacteria attachment and plaque build-up to reduce secondary caries. The protein-repellent method might be applicable to other dental materials.

International Journal of Oral Science (2015) 7, 103-109; doi:10.1038/ijos.2014.77; published 6 February 2015

Keywords: caries inhibition; human saliva microcosm biofilm; mechanical property; protein repellent; resin composite

\section{INTRODUCTION}

Dental caries, a dietary carbohydrate-modified bacterial infectious disease, is a common infection in humans. ${ }^{1}$ The basic mechanism of caries is demineralisation of the enamel and dentin via acid generated by a bacterial biofilm. ${ }^{2}$ Resin composites are increasingly being used for tooth cavity restorations because of their aesthetics and their direct-filling capability. ${ }^{3-4}$ Extensive efforts have improved resin compositions and curing conditions and have reduced polymerisation shrinkage. ${ }^{5-10}$ However, secondary caries and restorative material fractures represent more than $90 \%$ of recorded failures. ${ }^{11-12}$ Approximately one-half of all dental restorations fail within 10 years. ${ }^{13}$ The replacement of failed restorations has accounted for $50 \%-70 \%$ of all restorations performed. ${ }^{13}$ In addition, resin composites not only have no antibacterial properties, but they also can even accumulate more biofilm in vivo than other restorative materials. ${ }^{14-15}$ Therefore, it is desirable to improve the longevity of composite restorations by incorporating bioactive agents to combat microbial destruction and secondary caries while sustaining their load-bearing capability.

To reduce biofilm and plaque build-up and to combat caries, novel quaternary ammonium methacrylates were developed and incorporated into dental resins. ${ }^{16-18}$ 12-Methacryloyloxydodecylpyridinium bromide can be copolymerised with other dental monomers to form antibacterial polymer matrices to reduce bacterial growth. ${ }^{19-24}$ Other compositions, such as methacryloxylethylcetyl dimethyl ammonium chloride, have also been developed. ${ }^{25}$ Recently, quaternary ammonium dimethacrylate was synthesised and incorporated into bonding agents and composites to obtain antibacterial activity. ${ }^{26-30}$

One potential limitation of resins containing quaternary ammonium methacrylates is that the depositing of salivary proteins on composite surfaces could decrease the efficacy of 'contact inhibition',

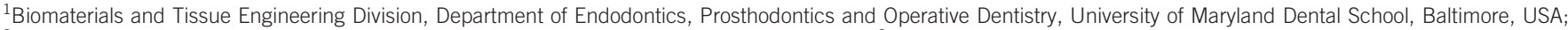

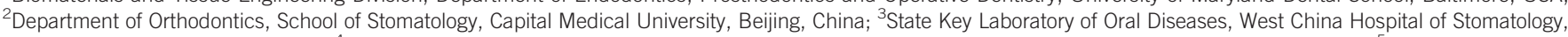

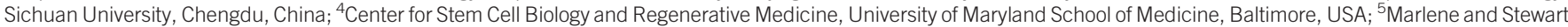

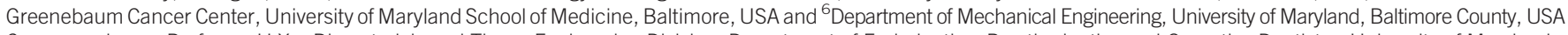
Correspondence: Professor H Xu, Biomaterials and Tissue Engineering Division, Department of Endodontics, Prosthodontics and Operative Dentistry, University of Maryland Dental School, Baltimore MD 21201, USA

E-mail: hxu@umaryland.edu

Dr L Cheng, State Key Laboratory of Oral Diseases, West China Hospital of Stomatology, Sichuan University, No.14, Section 3, Renmin South Road, Chengdu 610041, China E-mail: chengleidentist@163.com

Professor YX Bai, Department of Orthodontics, School of Stomatology, Capital Medical University, Beijing 100050, China

E-mail: byuxing@263.net

Accepted 8 October 2014 
thereby reducing antibacterial potency. ${ }^{31-32}$ Salivary proteins in the mouth can adhere to dental restoration surfaces to provide anchor points for bacteria attachment, an initial step in biofilm formation. ${ }^{33-34}$ Biofilm formation is a source of infection and a prerequisite for the occurrence of dental caries and secondary caries. ${ }^{35}$ Accordingly, there is a great need to develop a novel resin composite that can repel proteins. Such a composite could repel proteins and hence inhibit bacteria attachment, and it could further enhance the antibacterial potency of quaternary ammonium methacrylate-containing restorations by having a protein-repellent coating and enhancing the contact-killing efficacy. However, to date, there has been no report on dental composites that possess protein-repellent capability.

2-Methacryloyloxyethyl phosphorylcholine (MPC) is a methacrylate with a phospholipid polar group in the side chain, and it is a common biocompatible and hydrophilic biomedical polymer. ${ }^{36}$ MPC has been shown to have excellent ability to repel protein adsorption and prevent bacterial adhesion. ${ }^{37-38}$ Several medical devices containing MPC have been developed and used clinically, such as artificial blood vessels, implantable artificial hearts and artificial lungs. ${ }^{38-40}$ However, there have been no reports of the incorporation of MPC into dental composites.

Therefore, in this study, MPC was incorporated into a dental resin, which was then filled with glass particles to develop a protein-repellent composite for the first time. The objectives were (i) to develop a protein-repellent and bacteria-repellent dental composite and (ii) to investigate the effects of MPC mass fraction on the mechanical properties, protein adsorption and biofilm activity of the composite. The following hypotheses were tested: (i) MPC-containing composites would have mechanical properties matching those of a commercial control composite; (ii) MPC-containing composites would have much less protein adsorption on the composite surface than that without MPC; and (iii) MPC-containing composites would significantly reduce bacterial attachment and biofilm growth on the composite surface.

\section{MATERIALS AND METHODS}

\section{Fabrication of MPC-containing resin composites}

Bisphenol glycidyl dimethacrylate (BisGMA) and triethylene glycol dimethacrylate (TEGDMA) (Esstech, Essington, PA, USA) were mixed at a mass ratio of $1: 1$ and were rendered light-curable with $0.2 \%$ camphorquinone and $0.8 \%$ ethyl $4-\mathrm{N}, \mathrm{N}$-dimethylaminobenzoate (mass fractions). MPC, a methacrylate with a phospholipid polar group in the side chain, was used as the protein-repellent agent. The chemical structure of MPC, according to a previous study, ${ }^{36}$ is shown in Figure 1a. MPC was obtained from Sigma-Aldrich (St Louis, MO, USA) and was synthesized via the method reported by Ishihara et al. ${ }^{36}$ The MPC powder was mixed with photo-activated BisGMATEGDMA resin (referred to as BT) at the following MPC/ (BT+MPC) mass fractions: $0,2.5 \%, 5 \%, 7.5 \%, 10 \%, 15 \%$ and $20 \%$, yielding seven respective groups. The different mass fractions enabled the investigation of the relationship between the MPC mass fraction and mechanical properties of the composite. Barium boroaluminosilicate glass of a mean particle size of $1.4 \mu \mathrm{m}$ (Caulk/Dentsply, Milford, DE, USA) was silanised with 4\% 3-methacryloxypropyltrimethoxysilane and $2 \%$ n-propylamine. ${ }^{41}$ The glass particles were mixed into each resin at the same filler level of $70 \%$ by mass. Because the resin mass fraction was $30 \%$ in the composite, the MPC mass fractions in the composite were 0 (control), $0.75 \%, 1.5 \%, 2.25 \%$, $3.0 \%, 4.5 \%$ and $6.0 \%$ for the respective groups. As another control, a commercial composite with nanofillers of 40-200 nm was used (Heliomolar, Ivoclar, Ontario, Canada). The fillers consisted of silica and ytterbium-trifluoride at a filler mass fraction of $66.7 \%$. Heliomolar is indicated for Class I and II restorations in the posterior region, as well as Class III-V restorations. Eight composites were tested for mechanical properties:

(i) commercial control (Heliomolar);

(ii) $\quad 70 \%$ glass filler $+30 \%$ BisGMA-TEGDMA resin (termed 'Control without MPC');

(iii) $\quad 70 \%$ glass filler $+29.25 \%$ BisGMA-TEGDMA resin $+0.75 \%$ MPC ('0.75\% MPC');

(iv) $\quad 70 \%$ glass filler $+28.5 \%$ BisGMA-TEGDMA resin $+1.5 \% \mathrm{MPC}$ (' $1.5 \%$ MPC');

(v) $\quad 70 \%$ glass filler $+27.75 \%$ BisGMA-TEGDMA resin $+2.25 \%$ MPC ('2.25\% MPC');

(vi) $\quad 70 \%$ glass filler $+27 \%$ BisGMA-TEGDMA resin $+3 \%$ MPC ('3\% MPC');

a<smiles>C=C(C)C(=O)OCC[P+](=O)([O-])CC[NH2+][14CH3]</smiles>
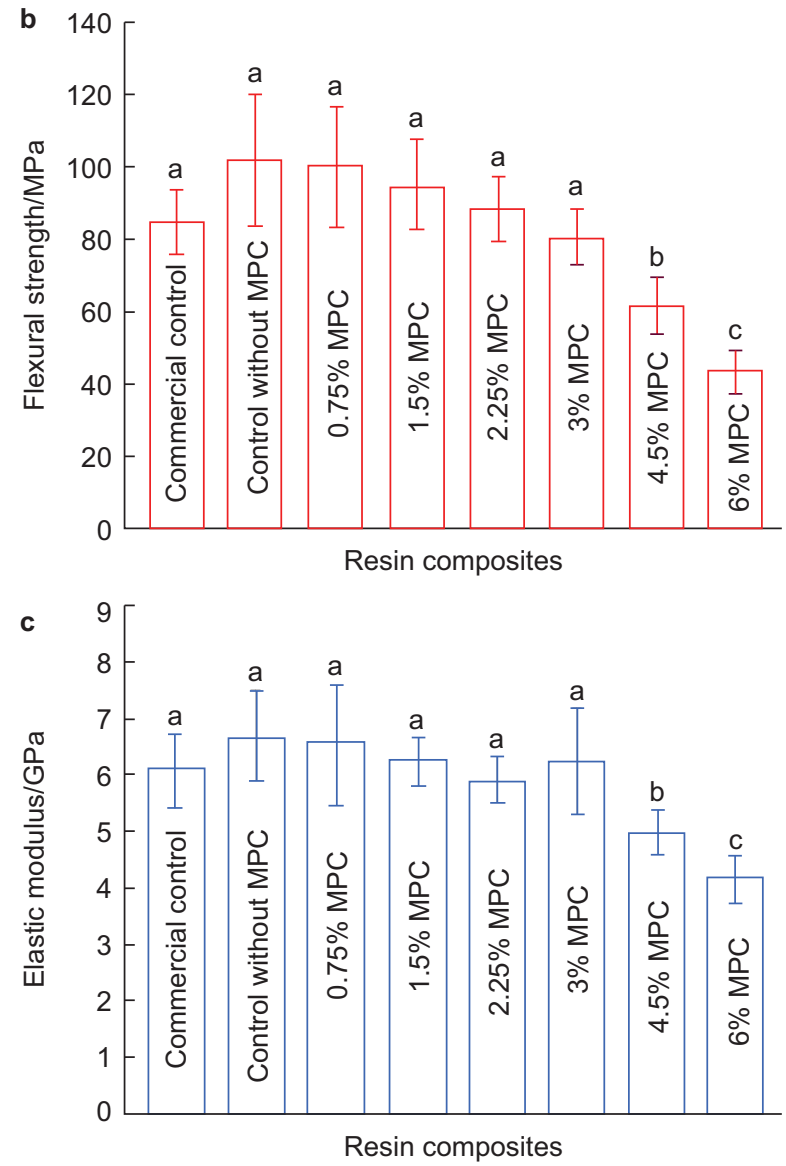

Figure 1 Mechanical properties of dental composites. (a) Chemical structure of MPC; (b) flexural strength and (c) elastic modulus of composites (mean \pm standard deviation; $n=6$ ). The composite specimens were immersed in distilled water at $37^{\circ} \mathrm{C}$ for $24 \mathrm{~h}$ and were then fractured while wet, within a few minutes after being removed from the water. Different letters indicate values that are significantly different from each other $(P<0.05)$. MPC, 2-methacryloyloxyethyl phosphorylcholine. 
(vii) $\quad 70 \%$ glass filler $+25.5 \%$ BisGMA-TEGDMA resin $+4.5 \% \mathrm{MPC}$ (' $4.5 \%$ MPC');

(viii) $70 \%$ glass filler $+24 \%$ BisGMA-TEGDMA resin $+6 \%$ MPC ('6\% MPC').

\section{Mechanical properties}

Each composite paste was placed into rectangular moulds of $2 \mathrm{~mm} \times 2 \mathrm{~mm} \times 25 \mathrm{~mm}$ and was photo-cured (Triad 2000; Dentsply, York, PA, USA) for $1 \mathrm{~min}$ on each open side. ${ }^{26-27}$ Six specimens per composite were made. A computer-controlled Universal Testing Machine (5500R; MTS, Cary, NC, USA) was used. ${ }^{26-27}$ Composite specimens were stored in distilled water at $37{ }^{\circ} \mathrm{C}$ for $24 \mathrm{~h}$ and then were fractured in three-point flexure with a $10 \mathrm{~mm}$ span at a crosshead speed of $1 \mathrm{~mm} \cdot \mathrm{min}^{-1} \cdot{ }^{26-27}$ The specimens were wet and not dried, and they were fractured within a few minutes after being removed from the water. Flexural strength $(S)$ was calculated as: $S=3 P_{\max } L /\left(2 b h^{2}\right)$, where $P$ is the fracture load, $L$ is span, $b$ is specimen width and $h$ is thickness. Elastic modulus $(E)$ was calculated as: $E=(P / d)\left(L^{3} /\left[4 b h^{3}\right]\right)$, where load $P$ divided by displacement $d$ is the slope in the linear elastic region. ${ }^{26-27}$

\section{Measurement of protein adsorption}

The mechanical results showed that group 7 with $4.5 \%$ MPC and group 8 with $6 \%$ MPC had relatively lower strength and elastic modulus values. Therefore, only groups $1-6$ were included in further protein and biofilm experiments. For the protein adsorption and biofilm experiments, each composite paste was placed into disc moulds of $9 \mathrm{~mm}$ in diameter and $2 \mathrm{~mm}$ in thickness. They were light-cured and stored in distilled water at $37{ }^{\circ} \mathrm{C}$ for $24 \mathrm{~h}$ as described above.

The amount of protein adsorbed on the composite discs was determined by the microbicinchoninic acid method. ${ }^{42-43}$ Each composite disc was immersed in phosphate-buffered saline (PBS) for $2 \mathrm{~h}$ before immersing in $4.5 \mathrm{~g} \cdot \mathrm{L}^{-1}$ bovine serum albumin (BSA) (Sigma-Aldrich, St Louis, MO, USA) solution at $37^{\circ} \mathrm{C}$ for $2 \mathrm{~h}$. The discs then were rinsed with fresh PBS by stirring at a speed of $300 \mathrm{r} \cdot \mathrm{min}^{-1}$ for $5 \mathrm{~min}$ (Bellco Glass, Vineland, NJ, USA), and they were immersed in $1 \%$ $(\mathrm{m} / \mathrm{m})$ sodium dodecyl sulphate in PBS and sonicated at room temperature for $20 \mathrm{~min}$ to detach completely the BSA adsorbed onto the surface of the disc. ${ }^{42-43}$ A protein analysis kit (micro bicinchoninic acid protein assay kit; Fisher Scientific, Pittsburgh, PA, USA) was used to determine the BSA concentration in the sodium dodecyl sulphate solution. Briefly, $25 \mu \mathrm{L}$ of the sodium dodecyl sulphate solution and $200 \mu \mathrm{L}$ of the bicinchoninic acid working reagent were mixed into the wells of a 96 -well plate and incubated at $60{ }^{\circ} \mathrm{C}$ for $30 \mathrm{~min} .{ }^{42-43}$ Then, the 96-well plate was cooled to room temperature, and the absorbance at $562 \mathrm{~nm}$ was measured via a microplate reader (SpectraMax M5; Molecular Devices, Sunnyvale, CA, USA). Standard curves were prepared using the BSA standard. From the concentration of protein, the amount of protein adsorbed on the disc surface was calculated. ${ }^{42-43}$

\section{Dental plaque microcosm biofilm model}

A dental plaque microcosm biofilm model was used. ${ }^{26-27}$ Saliva is ideal for growing microcosm biofilms in vitro, with the advantage of maintaining much of the complexity and heterogeneity of dental plaque in vivo. ${ }^{44}$ Saliva was collected from 10 healthy donors having natural dentition without active caries and not having used antibiotics within the preceding 3 months. The donors did not brush their teeth for $24 \mathrm{~h}$, and they abstained from food and drink intake for $2 \mathrm{~h}$ prior to donating saliva. ${ }^{26-27}$ Stimulated saliva was collected during paraffin chewing and was kept on ice. An equal volume of saliva from each of the ten donors was combined to form the saliva sample. The saliva was diluted in sterile glycerol to a concentration of $70 \%$ and was stored at $-80{ }^{\circ} \mathrm{C}$ for subsequent use. ${ }^{45}$

The saliva-glycerol stock was added, at a 1:50 final dilution, to the growth medium as an inoculum. The growth medium contained mucin (type II, porcine, gastric) at a concentration of $2.5 \mathrm{~g} \cdot \mathrm{L}^{-1}$, bacteriological peptone at $2.0 \mathrm{~g} \cdot \mathrm{L}^{-1}$, tryptone at $2.0 \mathrm{~g} \cdot \mathrm{L}^{-1}$, yeast extract at $1.0 \mathrm{~g} \cdot \mathrm{L}^{-1}, \mathrm{NaCl}$ at $0.35 \mathrm{~g} \cdot \mathrm{L}^{-1}, \mathrm{KCl}$ at $0.2 \mathrm{~g} \cdot \mathrm{L}^{-1}, \mathrm{CaCl}_{2}$ at $0.2 \mathrm{~g} \cdot \mathrm{L}^{-1}$, cysteine hydrochloride at $0.1 \mathrm{~g} \cdot \mathrm{L}^{-1}$, haemin at $0.001 \mathrm{~g} \cdot \mathrm{L}^{-1}$ and vitamin $\mathrm{K}_{1}$ at $0.0002 \mathrm{~g} \cdot \mathrm{L}^{-1}$, at $\mathrm{pH} 7 .{ }^{46}$ Composite discs were sterilised in ethylene oxide (Anprolene AN 74i; Andersen, Haw River, NC, USA). A volume of $1.5 \mathrm{~mL}$ of inoculum was added to each well of a 24-well plate with a disc followed by incubation at $37{ }^{\circ} \mathrm{C}$ in $5 \% \mathrm{CO}_{2}$ for $8 \mathrm{~h}$. Then, the discs were transferred to new 24 -well plates filled with fresh medium and incubated. After $16 \mathrm{~h}$, the discs were transferred to new 24 -well plates with fresh medium and were incubated for $24 \mathrm{~h}$. This process totalled $48 \mathrm{~h}$ of incubation, which was adequate to form plaque microcosm biofilms, as shown in previous studies. ${ }^{26-27}$

\section{Live/dead assay}

Two live/dead staining experiments were performed. First, saliva-glycerol stock was added, at a 1:50 final dilution, to the growth medium as an inoculum. Inoculum at a volume of $1.5 \mathrm{~mL}$ was added to each well of a 24-well plate containing a composite disc followed by incubation for $4 \mathrm{~h}$ to examine bacteria early attachment. ${ }^{47}$ Second, using a separate batch of composite discs, the culture lasted for $48 \mathrm{~h}$, as described in the section on 'Dental plaque microcosm biofilm model'.

After either $4 \mathrm{~h}$ or $48 \mathrm{~h}$ of growth, the microcosm biofilms on composite discs were gently washed three times with PBS and were stained using the BacLight live/dead kit (Molecular Probes, Eugene, OR, USA). Live bacteria were stained with Syto 9 to produce green fluorescence. Bacteria with compromised membranes were stained with propidium iodide to produce red fluorescence. The stained discs were examined using an inverted epifluorescence microscope (Eclipse TE2000-S; Nikon, Melville, NY, USA). The area of green staining (live bacteria) was computed with NIS Elements imaging software (Nikon, Melville, NY, USA). The area fraction of live bacteria= green staining area/total area of the image. Six composite discs were evaluated for each group at each time period. Three randomly chosen fields of view were photographed from each disc, yielding a total of 18 images for each condition.

\section{Biofilm colony-forming unit counts}

Discs with $48 \mathrm{~h}$ biofilms were transferred into tubes with $2 \mathrm{~mL}$ of cysteine peptone water (CPW), and the biofilms were harvested by sonication and vortexing (Fisher, Pittsburgh, PA, USA). ${ }^{26-27}$ Three types of agar plates were used to measure the colony-forming unit (CFU) counts and assess the microorganism viability. First, tryptic soy blood agar culture plates were used to determine total microorganisms. ${ }^{46}$ Second, mitis salivarius agar culture plates containing $15 \%$ sucrose were used to determine total Streptococci ${ }^{48}$ because mitis salivarius agar contains selective agents, including crystal violet, potassium tellurite and trypan blue, which inhibit most Gram-negative bacilli and most Gram-positive bacteria except for Streptococci, thus enabling the Streptococci to grow. ${ }^{48}$ Third, cariogenic Streptococcus mutans is known to be resistant to bacitracin, and this property has been used to isolate $S$. mutans from the highly heterogeneous oral microflora. ${ }^{46}$ Therefore, mitis salivarius agar culture plates plus 0.2 units of bacitracin per millilitre were used to determine S. mutans. ${ }^{46}$ The bacterial suspensions were serially diluted, spread onto agar plates and incubated at $37{ }^{\circ} \mathrm{C}$ in $5 \% \mathrm{CO}_{2}$ for $24 \mathrm{~h}^{26-27}$ The number of col- 
onies that grew were counted and used, along with the dilution factor, to calculate the total CFUs on each disc. ${ }^{26-27}$

\section{Statistical analysis}

One-way and two-way analyses of variance were performed to detect the significant effects of the variables. Tukey's multiple comparison test was used to compare the data at a $P$ value of 0.05 .

\section{RESULTS}

Figure 1 plots the flexural strength (Figure 1b) and elastic modulus (Figure 1c) of the composites (mean \pm standard deviation; $n=6$ ). The composites with $0.75 \%$ to $3 \%$ MPC had flexural strengths $(P=0.22)$ and elastic moduli $(P=0.37)$ similar to those of the commercial control and the control without MPC. Composites with 4.5\% MPC and $6 \%$ MPC had strengths and moduli that were significantly lower than those of the commercial control $(P<0.05)$.

The amounts of protein adsorption on composite disc surfaces are plotted in Figure 2 (mean \pm standard deviation; $n=6$ ). Adding MPC to composites significantly decreased the protein adsorption $(P<0.05)$. Protein adsorption was inversely proportional to the MPC mass fraction in the composite from 0 to 3\% MPC. The resin composite with $3 \%$ MPC had the lowest amount of protein adsorption, which was nearly $1 / 12$ that of the commercial control and the composite without $\operatorname{MPC}(P<0.05)$.

Bacterial early attachment onto composite surfaces was examined at $4 \mathrm{~h}$. Representative live/dead staining images at $4 \mathrm{~h}$ are shown in Figure $3 \mathrm{a}-3 \mathrm{~d}$, and the area fraction of composite surface covered by live bacteria is plotted in Figure $3 \mathrm{e}$ (mean \pm standard deviation; $n=6$ ). Live bacteria were stained green, and dead bacteria were stained red. The composite discs had primarily live bacteria, with few dead bacteria. The commercial control composite and the composite without MPC had noticeably more bacteria coverage than composites containing MPC. This outcome was found to be true and consistent by examining all 18 of the images per group. The composite with 3\% MPC had

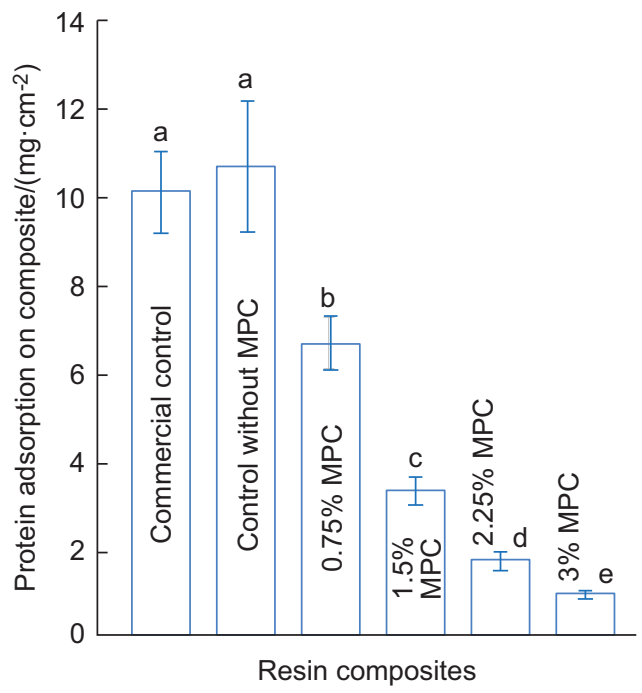

Figure 2 Protein adsorption onto composite surfaces. Mean \pm standard deviation; $n=6$. The composite with $3 \% \mathrm{MPC}$ had the lowest amount of protein adsorption, which was approximately $1 / 12$ those of the commercial composite control and the experimental composite without MPC $(P<0.05)$. Different letters indicate values that are significantly different from each other $(P<0.05)$. MPC, 2-methacryloyloxyethyl phosphorylcholine.

much less bacterial adhesion. For the quantification of live bacteria coverage in Figure 3e), values with different letters are significantly different from each other.

Figure 4 shows the results for 2-day biofilms on the composites. Relatively mature biofilms were formed in 2 days, covering nearly the entire surface of commercial composite and that without MPC (Figure $4 \mathrm{a}$ and $4 \mathrm{~b}$ ). However, there was less biofilm coverage on composite discs containing MPC (Figure $4 \mathrm{c}$ and $4 \mathrm{~d}$ ). In the quantification of the area fraction of live bacteria in Figure $4 \mathrm{e}$ (mean \pm standard
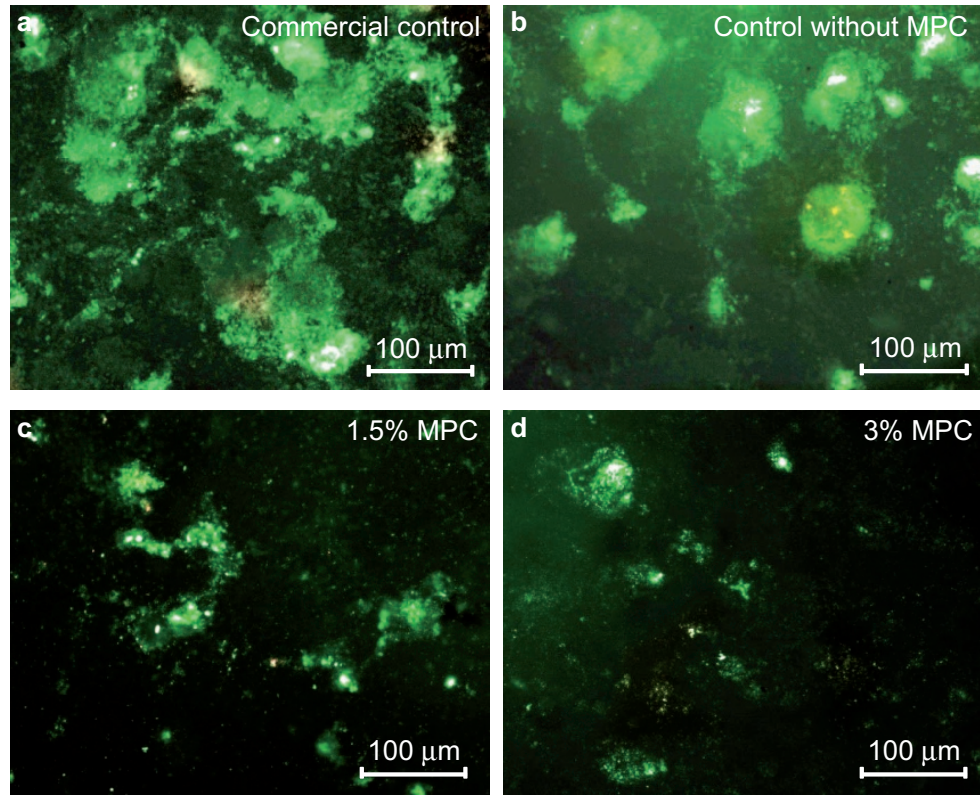

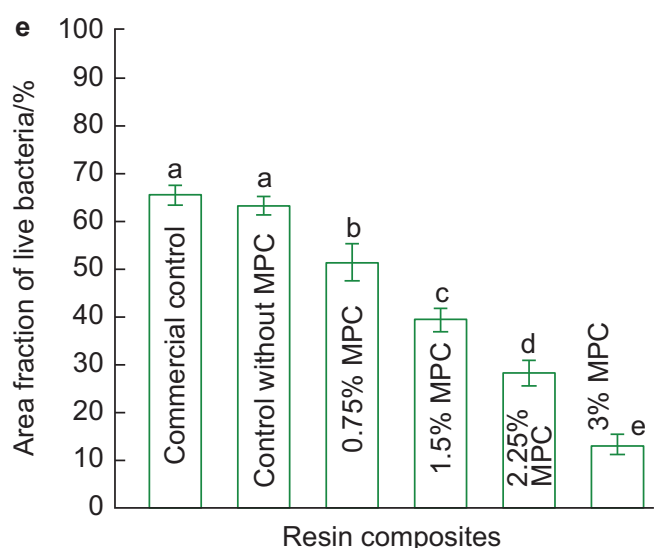

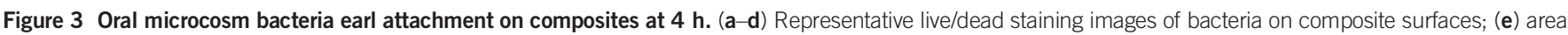

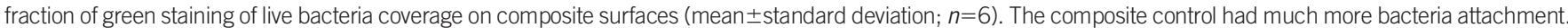
Increasing the MPC content decreased the bacterial attachment. Different letters in (e) indicate values that are significantly different from each other $(P<0.05)$. 

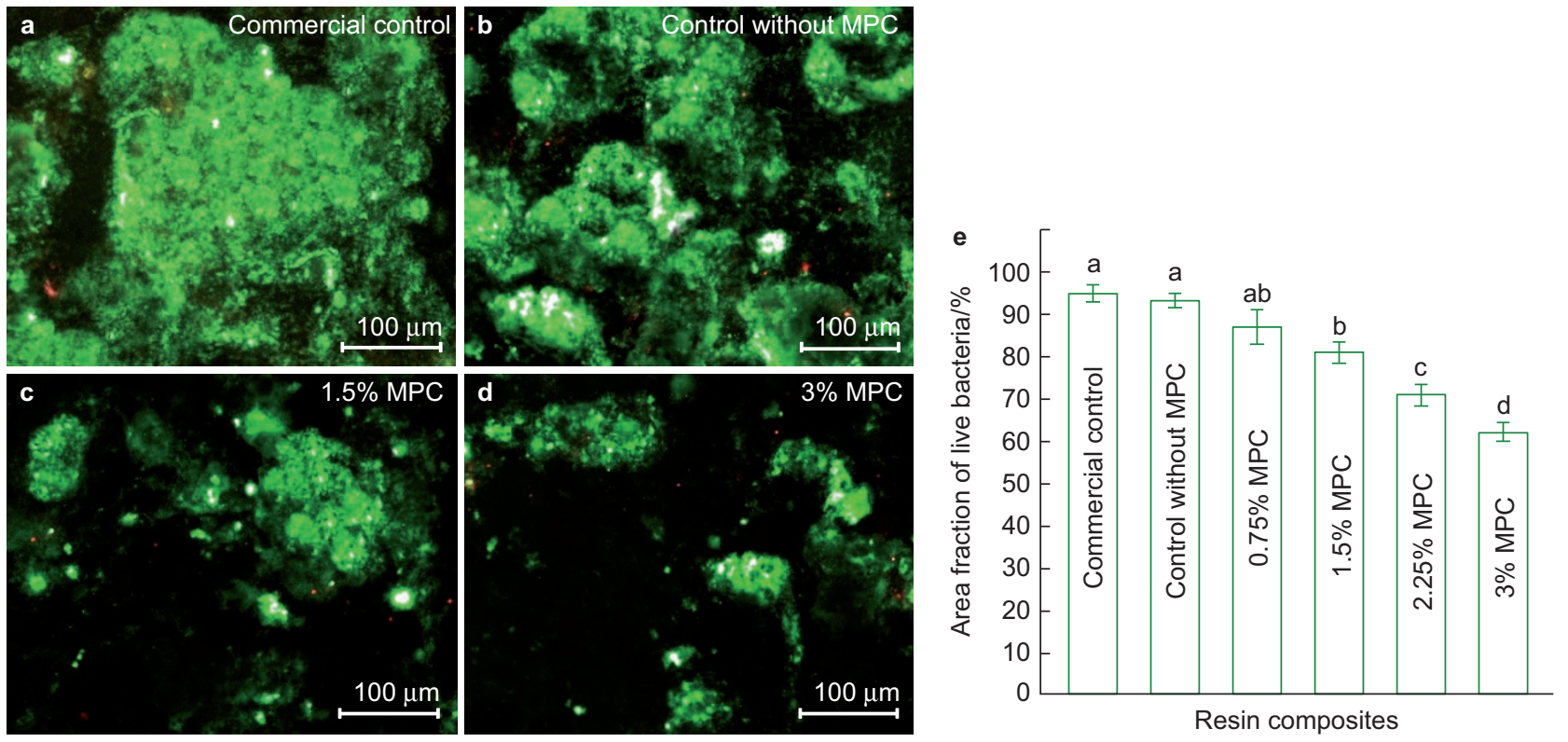

Figure 4 Oral microcosm biofilm growth on composites at $48 \mathrm{~h}$. (a-d) Representative live/dead staining images of biofilms on composite surfaces; (e) area fraction of green staining of live bacteria coverage on composite surfaces (mean \pm standard deviation; $n=6$ ). Increasing the MPC mass fraction decreased the biofilm coverage on the composite. The composite with 3\% MPC had the least biofilm coverage $(P<0.05)$. Different letters in $(\mathbf{e})$ indicate values that are significantly different from each other $(P<0.05)$.
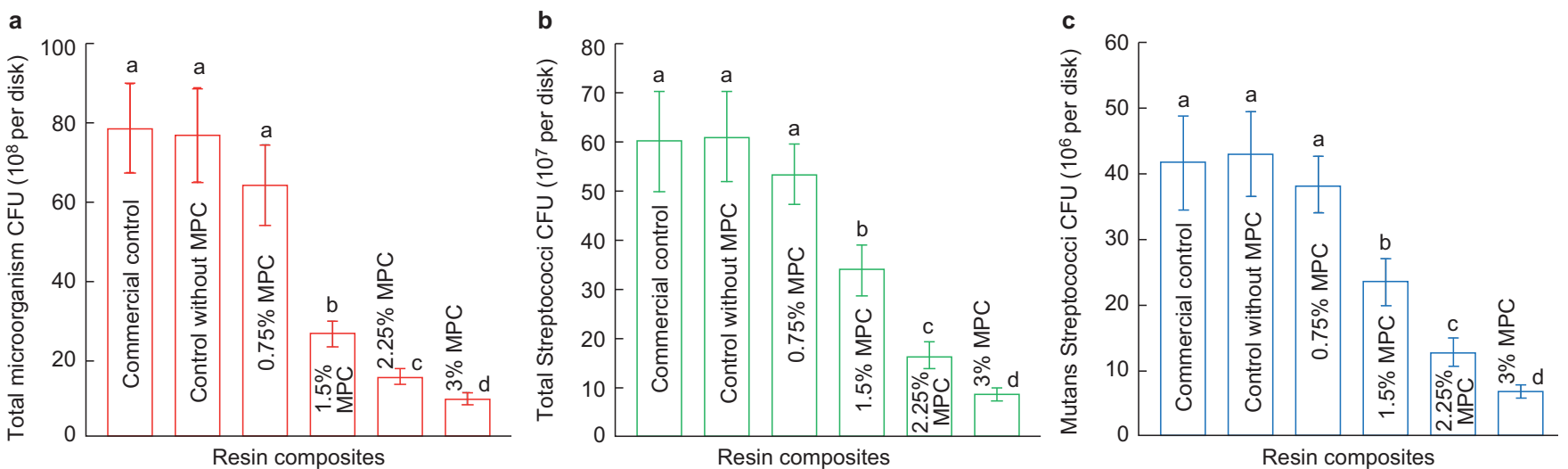

Figure 5 CFU counts of 2-day biofilms on composites. (a) Total microorganisms, (b) total Streptococci and (c) Streptococcus mutans (mean \pm standard deviation; $n=6$ ). Increasing the MPC mass fraction decreased the biofilm CFUs on the composites $(P<0.05)$. All three CFU counts on the composite with $3 \%$ MPC were much lower than those of the commercial composite control and the experimental composite without MPC $(P<0.05)$. In each plot, different letters indicate values that are significantly different from each other $(P<0.05)$. CFU, colony-forming unit; MPC, 2-methacryloyloxyethyl phosphorylcholine.

deviation; $n=6$ ), composites with $1.5 \%-3 \%$ MPC had significantly less biofilm coverage than the controls $(P<0.05)$.

Figure 5 plots the CFU counts of biofilms grown for 2 days on composite discs: total microorganisms (Figure 5a), total Streptococci (Figure 5b) and S. mutans (Figure 5c) (mean \pm standard deviation; $n=6$ ). The commercial control composite and the experimental composite without MPC had similarly high CFU counts $(P=0.89)$. All three CFU counts showed a decreasing trend with an increasing MPC mass fraction. All three CFU counts on the composite with 3\% MPC were greatly reduced compared to those of the controls $(P<0.05)$.

\section{DISCUSSION}

The present study represents the first report on the development of protein-repellent dental composite and investigation of the effects of
MPC incorporation on protein adsorption, biofilm activity, and the mechanical properties of the composite. MPC addition provided strong protein-repellent activity for the composite, and the microcosm bacteria early attachment $(4 \mathrm{~h})$ and mature biofilm (2 days) viability showed consistent and substantial decreases with increasing MPC mass fraction. The protein-repellent activity of the composite was achieved without compromising the mechanical properties of the composite from 0 to $3 \%$ of MPC. In addition, MPC had good biocompatibility, and it has already been used in several medical devices with approval of the Food and Drug Administration of the United States. ${ }^{39-40}$ Therefore, the novel protein-repellent approach is promising for developing dental composites to reduce bacteria attachment, biofilm and plaque build-up, and the occurrence of caries. Furthermore, this protein-repellent approach could be applied to 
develop a new class of protein-repellent dental resins, adhesives, cements, coatings and sealants.

There are two potential benefits of MPC-containing dental composite. First, salivary proteins adsorbed onto the resin composite surface in the oral environment provide a medium for the attachment of bacteria and microorganisms, thereby initiating the basis for biofilm formation. ${ }^{33-35}$ MPC has been shown to have excellent protein-repellent ability to diminish bacterial adhesion. ${ }^{37-38}$ Regarding the proteinrepellent mechanism, ${ }^{37,49-51}$ it was suggested that MPC is highly hydrophilic, ${ }^{36}$ and there is an abundance of free water but no bound water in the hydrated MPC polymer. The presence of bound water causes protein adsorption. ${ }^{37,50-51}$ By contrast, the large amount of free water around the phosphorylcholine group is believed to detach proteins effectively, thereby repelling protein adsorption. ${ }^{37,51}$ Based on this mechanism, it could be assumed that increasing the mass fraction of MPC in the resin composite would increase the presence of MPC and hence its protein-repellent potency. Indeed, in the present study, gradually increasing the MPC mass fraction from 0 to $3 \%$ in the composite significantly and monotonically decreased the amount of protein adsorption (Figure 3). That the MPC-containing composite could repel proteins indicated that the composite could potentially also reduce biofilm attachment. Indeed, the results in Figures 4-6 confirmed that the incorporation of MPC at a mass fraction of 3\% into the composite greatly reduced bacteria attachment, biofilm growth and CFU counts.

Second, previous studies have suggested that salivary proteins adsorbed from physiological fluids are able to attenuate significantly the antibacterial properties of the underlying surfaces. ${ }^{31-32}$ Protein adsorption onto the resin surface rendered the contact-killing mechanism less effective. Indeed, several studies have demonstrated that a saliva-derived protein film on the cationic antibacterial surface reduced the original bactericidal effect. ${ }^{52-54}$ For example, 12-methacryloyloxydodecylpyridinium bromide-immobilized fillers, which contained 12-methacryloyloxydodecylpyridinium bromide at a concentration of $15.8 \%$, greatly inhibited bacterial growth; however, saliva pretreatment of the surface reduced its antibacterial potency. ${ }^{54}$ Therefore, the reduction in the antibacterial effect by the adsorption of salivary proteins on the composite has been considered a drawback of dental materials with contact-killing antibacterial activities. Because the MPC-containing composite greatly reduced protein adsorption, it could likely enhance the antibacterial effects of contact-killing dental resins. Further study is needed to combine MPC with contact-killing antibacterial agents in the resin composite in order to investigate their synergistic effects on the antibacterial potency.

The present study used an artificial saliva-like culture medium (the McBain medium) for the biofilm culture experiments. A major component of this medium, mucin, accounts for up to $26 \%$ of the natural salivary proteins; mucin is an important salivary protein in the salivary pellicle. ${ }^{55-56}$ A recent study investigated the effects of salivary pellicle pre-coating on resin surfaces on the antibacterial properties of the resin. ${ }^{53}$ When cultured in McBain medium, there was no significant difference in antibacterial activity whether the resin specimens were precoated with salivary pellicles or not. These results suggested that the McBain artificial saliva medium produced medium-derived pellicles on the resin surfaces, which provided attenuating effects on biofilms similar to natural salivary pellicles. Hence, the present study used the McBain artificial saliva medium to test the bacteria attachment and biofilm growth on the MPC-containing composite, which better simulated the oral environment than using a culture medium such as water.
In addition to secondary caries due to biofilm acids, bulk fracture is also a main challenge facing composite restorations. ${ }^{11-12}$ Therefore, the new protein-repellent composite must possess loadbearing capability for tooth cavity restorations. In the present study, composites with MPC at up to $3 \%$ did not significantly decrease the composite strength, whereas $4.5 \%$ and $6 \%$ MPC decreased the mechanical properties. Further studies should investigate composite water sorption as a function of MPC mass fraction and determine whether the decrease in mechanical properties at $\geqslant 4.5 \% \mathrm{MPC}$ was due to an increase in the water sorption of the composite. ${ }^{57}$ Nonetheless, the present study showed the promise of using an optimal MPC mass fraction in the composite to achieve the maximal protein-repellent capability without significantly compromising the mechanical properties. In the present study, incorporating MPC into the composite at $0.75 \%-3 \% \mathrm{MPC}$ resulted in mechanical properties similar to those of a commercial composite used for Class I-IV restorations. The strength and elastic modulus of the composite containing 3\% MPC were also similar to those of the glass-filled composite without MPC. This finding indicated that significant protein-repellent ability could be achieved in dental composite without compromising the load-bearing capability compared to the counterpart composite without MPC. Therefore, the protein-repellent method with MPC incorporation into dental composite is promising in its potential to yield new composites for repelling protein adsorption and hence bacteria attachment.

Although it is important to inhibit bacterial growth and to possess load-bearing capability, it is equally important for a new composite to be non-cytotoxic to mammalian cells. Although many medical devices containing MPC have been developed and used clinically, ${ }^{38-40}$ there have been no reports on the incorporation of MPC into dental composites. Therefore, assessment of the cytotoxic behaviour of MPCcontaining composite must be performed in future studies. In addition, it should be noted that, although the MPC-containing composite could reduce bacterial adhesion, it had no antibacterial function, did not kill bacteria, and had no remineralisation capability. Further studies are needed to incorporate antibacterial and remineralisation agents into the MPC-containing dental composite to inhibit biofilm formation more effectively and to combat secondary caries.

\section{CONCLUSIONS}

In this study, the first protein-repellent dental composite was reported, and the effects of MPC mass fraction on the protein adsorption, biofilm activity, and mechanical properties of the composite were determined. Incorporation of MPC up to 3\% into the composite did not compromise the strength and elastic modulus, which matched those of a commercial composite without protein-repellent ability. Increasing the MPC mass fraction monotonically decreased protein adsorption, dental microcosm bacteria early attachment and biofilm growth. Biofilm CFU counts for total microorganisms, total streptococci, and mutans streptococci on composite with $3 \%$ MPC were greatly reduced compared to the controls. The MPC-containing composite is promising for the reduction of biofilm formation and the combatting of secondary caries. The protein-repellent method could have applicability to other composites, adhesives, sealants and cements to repel proteins and inhibit oral bacteria attachment.

\section{ACKNOWLEDGEMENTS}

We thank Dr Michael D. Weir, Dr Junling Wu and Prof Xuedong Zhou for their fruitful discussions and help. This study was supported by the School of Stomatology at the Capital Medical University in China (Ning Zhang), NIH 
R01 DE17974 (Hockin HK Xu) and by a Seed Grant (Hockin HK Xu) from the University of Maryland School of Dentistry.

1 Hu DY, Hong X, Li X. Oral health in China-trends and challenges. Int J Oral Sci2011; 3(1): 7-12.

2 Cenci MS, Pereira-Cenci T, Cury JA et al. Relationship between gap size and dentine secondary caries formation assessed in a microcosm biofilm model. Caries Res 2009, 43(2): 97-102.

3 Drummond JL. Degradation, fatigue, and failure of resin dental composite materials. J Dent Res 2008; 87(8): 710-719.

4 Ferracane JL. Resin composite - state of the art. Dent Mater 2011; 27(1): 29-38.

5 Lim BS, Ferracane JL, Sakaguchi RL et al. Reduction of polymerization contraction stress for dental composites by two-step light-activation. Dent Mater 2002; 18(6): 436-444.

6 Watts DC, Marouf AS, Al-Hindi AM. Photo-polymerization shrinkage-stress kinetics in resin-composites: methods development. Dent Mater 2003; 19(1): 1-11.

7 Lu H, Stansbury JW, Bowman CN. Impact of curing protocol on conversion and shrinkage stress. J Dent Res 2005; 84(9): 822-826.

$8 \mathrm{Xu} \mathrm{X}$, Ling L, Wang $\mathrm{R}$ et al. Formulation and characterization of a novel fluoridereleasing dental composite. Dent Mater 2006; 22(11): 1014-1023.

9 Wan Q, Sheffield J, McCool J et al. Light curable dental composites designed with colloidal crystal reinforcement. Dent Mater 2008; 24(12): 1694-1701.

10 Wei YJ, Silikas N, Zhang ZT et al. Hygroscopic dimensional changes of self-adhering and new resin-matrix composites during water sorption/desorption cycles. Dent Mater 2011; 27(3): 259-266.

11 Sakaguchi RL. Review of the current status and challenges for dental posterior restorative composites: clinical, chemistry, and physical behavior considerations. Summary of discussion from the Portland Composites Symposium (POCOS) June 17-19, 2004, Oregon Health and Science University, Portland, Oregon. Dent Mater 2005; 21(1): 3-6.

12 Sarrett DC. Clinical challenges and the relevance of materials testing for posterior composite restorations. Dent Mater 2005; 21(1): 9-20.

13 Deligeorgi V, Mjör IA, Wilson NH. An overview of reasons for the placement and replacement of restorations. Prim Dent Care 2001; 8(1): 5-11.

14 Zalkind MM, Keisar O, Ever-Hadani P et al. Accumulation of Streptococcus mutans on light-cured composites and amalgam: an in vitro study. J Esthet Dent 1998; 10(4) 187-190.

15 Beyth N, Domb AJ, Weiss El. An in vitro quantitative antibacterial analysis of amalgam and composite resins. J Dent 2007; 35(3): 201-206.

16 Antonucci JM, Zeiger DN, Tang $\mathrm{K}$ et al. Synthesis and characterization of dimethacrylates containing quaternary ammonium functionalities for dental applications. Dent Mater 2012; 28(2): 219-228.

17 Weng $\mathrm{Y}$, Howard L, Guo $\mathrm{X}$ et al. A novel antibacterial resin composite for improved dental restoratives. J Mater Sci Mater Med 2012; 23(6): 1553-1561.

$18 \mathrm{Xu} \mathrm{X}$, Wang Y, Liao $\mathrm{S}$ et al. Synthesis and characterization of antibacterial dental monomers and composites. J Biomed Mater Res Part B Appl Biomater 2012; 100(4): 1151-1162.

19 Imazato S. Bio-active restorative materials with antibacterial effects: new dimension of innovation in restorative dentistry. Dent Mater J 2009; 28(1): 11-19.

20 Imazato S. Antibacterial properties of resin composites and dentin bonding systems. Dent Mater 2003; 19(6): 449-457.

21 Imazato S, Tay FR, Kaneshiro AV et al. An in vivo evaluation of bonding ability of comprehensive antibacterial adhesive system incorporating MDPB. Dent Mater 2007: 23(2): 170-176.

22 Imazato S, Ehara A, Torii M et al. Antibacterial activity of dentine primer containing MDPB after curing. J Dent 1998; 26(3): 267-271.

23 Imazato $\mathrm{S}$, Kinomoto $\mathrm{Y}$, Tarumi $\mathrm{H}$ et al. Antibacterial activity and bonding characteristics of an adhesive resin containing antibacterial monomer MDPB. Dent Mater 2003; 19(4): 313-319.

24 Zhang K, Cheng L, Imazato $\mathrm{S}$ et al. Effects of dual antibacterial agents MDPB and nano-silver in primer on microcosm biofilm, cytotoxicity and dentine bond properties. J Dent 2013; 41(5): 464-474.

25 Li F, Chen J, Chai Z et al. Effects of a dental adhesive incorporating antibacterial monomer on the growth, adherence and membrane integrity of Streptococcus mutans. J Dent 2009; 37(4): 289-296.

26 Cheng L, Weir MD, Xu HH et al. Antibacterial amorphous calcium phosphate nanocomposites with a quaternary ammonium dimethacrylate and silver nanoparticles. Dent Mater 2012; 28(5): 561-572.

27 Cheng L, Weir MD, Zhang $\mathrm{K}$ et al. Dental plaque microcosm biofilm behavior on calcium phosphate nanocomposite with quaternary ammonium. Dent Mater 2012; 28(8): 853-862.

28 Cheng L, Weir MD, Zhang $\mathrm{K}$ et al. Antibacterial nanocomposite with calcium phosphate and quaternary ammonium. J Dent Res 2012; 91(5): 460-466.

29 Zhou C, Weir MD, Zhang K et al. Synthesis of new antibacterial quaternary ammonium monomer for incorporation into CaP nanocomposite. Dent Mater 2013; 29(8): 859-870.
30 Zhang K, Melo MA, Cheng $L$ et al. Effect of quaternary ammonium and silver nanoparticle-containing adhesives on dentin bond strength and dental plaque microcosm biofilms. Dent Mater 2012; 28(8): 842-852.

31 Beyth N, Yudovin-Farber I, Bahir R et al. Antibacterial activity of dental composites containing quaternary ammonium polyethylenimine nanoparticles against Streptococcus mutans. Biomaterials 2006; 27(21): 3995-4002.

32 Namba N, Yoshida Y, Nagaoka N et al. Antibacterial effect of bactericide immobilized in resin matrix. Dent Mater 2009; 25(4): 424-430.

33 Kolenbrander PE, London J. Adhere today, here tomorrow: oral bacterial adherence. J Bacteriol 1993; 175(11): 3247-3252.

34 Donlan RM, Costerton JW. Biofilms: survival mechanisms of clinically relevant microorganisms. Clin Microbiol Rev 2002; 15(2): 167-193.

35 Busscher HJ, Rinastiti M, Siswomihardjo W et al. Biofilm formation on dental restorative and implant materials. J Dent Res 2010; 89(7): 657-665.

36 Ishihara K, Ueda T, Nakabayashi N. Preparation of phospholipid polymers and their properties as polymer hydrogel membranes. Polym J 1990; 22(5): 355-360.

37 Ishihara K, Nomura H, Mihara T et al. Why do phospholipid polymers reduce protein adsorption? J Biomed Mater Res 1998; 39(2): 323-330.

38 Sibarani J, Takai M, Ishihara K. Surface modification on microfluidic devices with 2 methacryloyloxyethyl phosphorylcholine polymers for reducing unfavorable protein adsorption. Colloids Surf B Biointerfaces 2007; 54(1): 88-93.

39 Kuiper KK, Nordrehaug JE. Early mobilization after protamine reversal of heparin following implantation of phosphorylcholine-coated stents in totally occluded coronary arteries. Am J Cardiol 2000; 85(6): 698-702.

40 Lewis AL, Tolhurst LA, Stratford PW. Analysis of a phosphorylcholine-based polymer coating on a coronary stent pre- and post-implantation. Biomaterials 2002; 23(7): $1697-1706$

$41 \mathrm{Xu} \mathrm{HH}$, Moreau JL, Sun L et al. Nanocomposite containing amorphous calcium phosphate nanoparticles for caries inhibition. Dent Mater 2011; 27(8): 762-769.

42 Ishihara K, Ziats NP. Tierney BP et al. Protein adsorption from human plasma is reduced on phospholipid polymers. J Biomed Mater Res 1991; 25(11): 1397-1407.

43 Moro T, Kawaguchi $\mathrm{H}$, Ishihara $\mathrm{K}$ et al. Wear resistance of artificial hip joints with poly(2-methacryloyloxyethyl phosphorylcholine) grafted polyethylene: comparisons with the effect of polyethylene cross-linking and ceramic femoral heads. Biomaterials 2009; 30(16): 2995-3001.

44 McBain AJ. Chapter 4: In vitro biofilm models: an overview. Adv App/ Microbiol2009, 69: 99-132.

45 Cheng L, Exterkate RA, Zhou X et al. Effect of Galla chinensis on growth and metabolism of microcosm biofilms. Caries Res 2011; 45(2): 87-92.

46 McBain AJ, Sissons C, Ledder RG et al. Development and characterization of a simple perfused oral microcosm. J Appl Microbiol 2005; 98(3): 624-634.

47 Zhou H, Li F, Weir MD et al. Dental plaque microcosm response to bonding agents containing quaternary ammonium methacrylates with different chain lengths and charge densities. J Dent 2013; 41(11): 1122-1131.

48 Lima JP, Sampaio de Melo MA, Borges FM et al. Evaluation of the antimicrobial effect of photodynamic antimicrobial therapy in an in situ model of dentine caries. Eur J Oral Sci 2009; 117(5): 568-574.

49 Kyomoto M, Moro T, Miyaji $\mathrm{F}$ et al. Effects of mobility/immobility of surface modification by 2-methacryloyloxyethyl phosphorylcholine polymer on the durability of polyethylene for artificial joints. J Biomed Mater Res A 2009; 90(2): 362-371.

50 Tateishi T, Kyomoto M, Kakinoki $S$ et al. Reduced platelets and bacteria adhesion on poly(ether ether ketone) by photoinduced and self-initiated graft polymerization of 2-methacryloyloxyethyl phosphorylcholine. J Biomed Mater Res A 2014; 102(5): 1342-1349

51 Goda T, Konno T, Takai M et al. Photoinduced phospholipid polymer grafting on Parylene film: advanced lubrication and antibiofouling properties. Colloids Surf $B$ Biointerfaces 2007; 54(1): 67-73.

52 Müller R, Eidt A, Hiller KA et al. Influences of protein films on antibacterial or bacteriarepellent surface coatings in a model system using silicon wafers. Biomaterials 2009; 30(28): 4921-4929.

$53 \mathrm{Li} \mathrm{F}$, Weir MD, Fouad AF et al. Effect of salivary pellicle on antibacterial activity of novel antibacterial dental adhesives using a dental plaque microcosm biofilm model. Dent Mater 2014; 30(2): 182-191.

54 Imazato S, Ebi N, Takahashi Y et al. Antibacterial activity of bactericide-immobilized filler for resin-based restoratives. Biomaterials 2003; 24(20): 3605-3609.

55 Lendenmann U, Grogan J, Oppenheim FG. Saliva and dental pellicle-a review. Adv Dent Res 2000; 14: 22-28.

56 Amerongen AV, Bolscher JG, Veerman EC. Salivary mucins: protective functions in relation to their diversity. Glycobiology 1995; 5(8): 733-740.

57 Tay FR, Pashley DH. Water treeing - a potential mechanism for degradation of dentin adhesives. Am J Dent 2003; 16(1): 6-12.

This work is licensed under a Creative Commons Attribution-

NonCommercial-NoDerivs 3.0 Unported License. The images or other third party material in this article are included in the article's Creative Commons license, unless indicated otherwise in the credit line; if the material is not included under the Creative Commons license, users will need to obtain permission from the license holder to reproduce the material. To view a copy of this license, visit http://creativecommons.org/licenses/

by-nc-nd/3.0/ 